\title{
Endocrinology and treatment of trophoblastic tumours
}

\author{
K. D. BAGSHAWE
}

From the Department of Medical Oncology, Charing Cross Hospital, Fulham Palace Road, London

I shall confine my discussion of the endocrinology of trophoblastic tumours largely to those aspects which concern screening, diagnosis and the monitoring of therapy and follow up.

The endocrine repertoire of the trophoblast is well known to change sequentially during the course of pregnancy. In general, the endocrinological behaviour of choriocarcinoma resembles that of early pregnancy trophoblast rather than that of late pregnancy. Thus, synthesis of human chorionic gonadotrophin has been detectable in urine or plasma from all 500 cases with active malignant trophoblastic tumour studied at Charing Cross Hospital. On the contrary, human placental lactogen production, which is characteristically present in high concentration in the last trimester of pregnancy, was at a relatively low level and sometimes undetectable even before treatment was started (figure). Similarly,

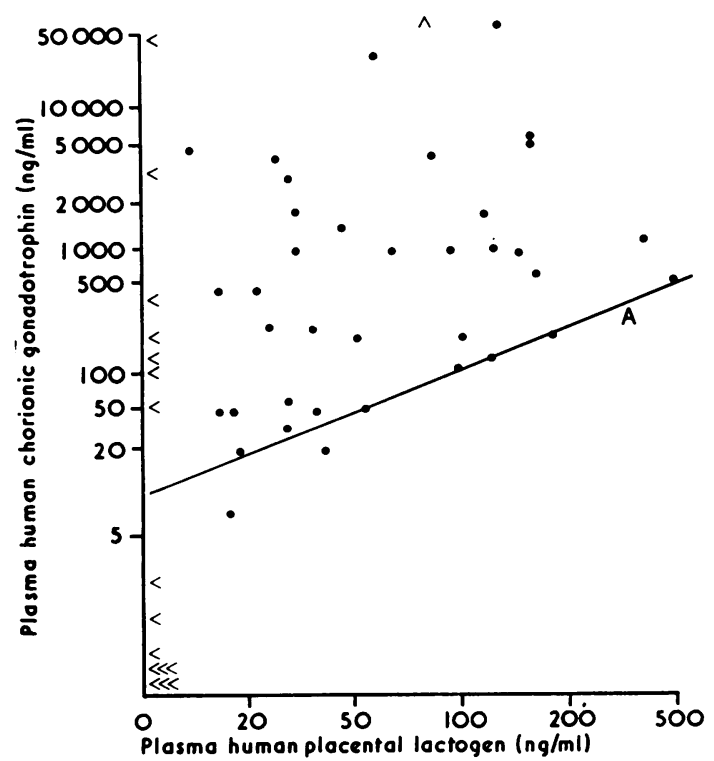

Fig Human chorionic gonadotrophin and corresponding human placental lactogen concentrations in patients with trophoblastic tumours. Line A indicates equivalent values. we have observed that the placental isoenzyme of alkaline phosphatase was not increased in the 35 patients we studied (16 gestational choriocarcinoma, seven testicular choriocarcinoma, three ovarian choriocarcinoma, nine invasive mole) (Jacoby, 1972; Jacoby and Bagshawe, 1972). $\alpha$-Fetoprotein $(\alpha \mathrm{FP})$ has been in the normal range in most patients with gestational choriocarcinoma and invasive mole, but elevated values were found in patients with hepatic metastases. $\alpha$ FP values are also low in hydatidform mole unless there is an associated fetus (Seppälä et al, 1972; Ishiguro, 1975).

Another pregnancy associated $\beta_{1}$ glycoprotein variously identified as ' $\mathrm{SP}_{1}$ ' (Bohn, 1974) or Papp-C (Lin et al, 1974) has been identified in the serum of patients with trophoblastic tumours (Tatarinov et al, 1974).

Urinary oestrogen excretion rates in women with mole were found to be generally above normal values in non-pregnant women but to be lower than those of women at a corresponding stage of normal pregnancy and they varied widely (Brinck-Johnsen $e t$ $a l, 1970)$. These workers found, and this is also our experience, that in patients with choriocarcinoma, oestrogen production was in the normal range for the non-pregnant or only marginally elevated. Serum progesterone values were elevated in most patients with metastatic disease and, with extensive disease, reached those obtained during pregnancy (Dawood, 1975). As an index of tumour activity it was, however, thought inferior to hCG, even when this was measured by a haemagglutination method. Plasma testosterone has been reported to be elevated, particularly in patients whose ovaries were enlarged (Samaan et al, 1972). The testosterone content of ovarian cyst fluid suggested that its major source was the ovary and a later study has shown that androstenedione and dihydrotesterone are increased in similar cases (Samaan et al, 1973).

In our experience with approximately 500 cases of invasive mole and choriocarcinoma, overt hyperthyroidism has been present in about $1 \%$. It is, however, relatively common in patients with hydatidiform mole (Kock et al, 1966). Increased thyroid 
function without clinical hyperthyroidism may also be observed (Odell et al, 1963). Evidence that human chorionic thyrotropin (HCT) is distinct from pituitary thyroid stimulating hormone (TSH) and that the thyroid stimulator in hydatidiform mole is a distinct entity, at least immunologically, has been presented (Hershman et al, 1970). More recently the same group have presented evidence suggesting that the thyrotropin of hydatidiform mole is hCG (Kenimer et al, 1975).

Measurements of hCG in urine, plasma and CSF provide a valuable 'marker' for these tumours. One important application is the screening of cases for treatment after hydatidiform mole. This is probably the first biochemical screening for cancer to come into clinical use and it is used to avoid treating unnecessarily those patients whose invasive moles undergo spontaneous degeneration, whilst ensuring that patients requiring treatment are identified at a stage and time when their tumours can be treated successfully (Bagshawe et al, 1969, 1973). This policy is, I think, preferable to the use of prophylactic chemotherapy for all cases of hydatidiform mole both with respect to potential fatality from tumour and with respect to potential teratogenicity.

Urinary hCG has been monitored by radioimmunoassay, after evacuation of hydatidiform mole in the UK since January 1972 under arrangements supported by the Department of Health and Social Security and the Royal College of Obstetricians and Gynaecologists. Examination of the data obtained so far has revealed that, of 520 patients not receiving oral contraceptives (or not confirming that they used them) $8.6 \%$ required chemotherapy; of 91 patients who started oral contraceptives before their hCG/ LH values had returned to normal $17.6 \%$ required chemotherapy (Stone et al, 1976).

It may take up to six months for gonadotrophin values to return to normal after hydatidiform mole and when this is achieved spontaneously it is generally a good prognostic sign. However, we have now seen four patients out of approximately 1200 followed up who have developed choriocarcinomas without new conceptions being evident; hCG/LH values had been normal for two to 12 months. There is also a small group of patients who, having had a mole and subsequently one or more pregnancies, then developed choriocarcinoma. Details of these observations will be published elsewhere.

The isolation of the distinctive $\beta$ subunit of hCG has resulted in the development and automation of a radioimmunoassay (Kardana and Bagshawe, 1976) which discriminates between hCG and $\mathrm{LH}$ and which as a result of this improved specificity, is 10 to 100 times more sensitive. At present, assay of $\beta$-hCG is performed only on plasma and it is especially valuable in monitoring therapy and in following up treated cases. However, for following up patients after hydatidiform mole the convenience of urinary assays remains important.

The identification of hCG in the body fluids of patients who have not had a hydatidform mole evacuated in the previous six months has, in my experience, always been indicative of cancer. With the use of sensitive $\beta$-hCG assays hCG production by non-trophoblastic tumours has been identified more frequently (Braunstein et al, 1973). Thus, hCG production by the non-pregnant subject should be assumed to be associated with a trophoblastic tumour until proven otherwise.

Since occult choriocarcinoma may cause cerebral haemorrhage, gastrointestinal bleeding, pulmonary hypertension, pleuritic pain, hepatomegaly and haematuria (Magrath et al, 1971), greater use of $\beta$ hCG assays in these conditions could avoid unnecessary and sometimes undesirable surgery.

Observations on a large number of patients in our series (Bagshawe, 1969) and elsewhere indicates a broad correlation between the amount of tumour and both plasma and urinary hCG values. It has been estimated that, on average, choriocarcinoma cells produce of the order of $5 \times 10^{-5} \mathrm{IU}$ hCG, ie, about $1 \mathrm{pg}$ per day so that with hCG assay detecting $1 \mathrm{~m}$ IU $(50 \mathrm{pg}) / \mathrm{ml}$ and a urinary clearance rate of $1 \mathrm{ml} /$ min the minimum viable tumour mass detectable is now of the order of $10^{4}$ to $10^{5}$ cells. (The minimum detected by pregnancy test is of the order $10^{8}$ to $10^{9}$ cells.) Whilst this is much smaller than the minimum detectable mass for any other tumour, cell residues which are undetectable by the most sensitive methods after chemotherapy are still capable of causing recurrent growth. Treatment is therefore continued after hCG has become undectable. The length of time for which we continue treatment is determined by the rate of response of the tumour as indicated by hCG values and by a consideration of the prognostic factors considered later.

Metastases to the central nervous system remain one of the principal causes of death and those which develop during chemotherapy are particularly lethal. Studies have shown that in the absence of brain metastases the plasma/CSF ratio for hCG is $>64 / 1$. Whereas in those with brain metastases it is almost invariably <64/1 (Rushworth et al, 1968; Bagshawe et al, 1968; Bagshawe and Harland, 1976) and regular determinations can be used for presymptomatic detection and monitoring.

The introduction of methotrexate for the treatment of trophoblastic tumours ( $\mathrm{Li}$ et al, 1956) was one of the decisive events in the development of anti-cancer chemotherapy. Since then it has become clear that this agent used alone is capable of eradicating totally 
some of these potentially fatal tumours. But it is important to recognize that whilst it rarely fails to eradicate invasive moles when used as a single agent, in choriocarcinoma it produces a therapeutic response in about $70 \%$ and is curative in only about $25 \%$ of cases. Some methotrexate-resistant patients were found to respond to actinomycin D (Ross et al, 1962) but combination chemotherapy was introduced early in an attempt to avoid drug resistance (Bagshawe and McDonald, 1960).

Ideally it should be possible to compare different regimens of chemotherapy from published accounts of patients treated. Unfortunately, this is not so. One of the principal difficulties in assessing progress in the treatment of these tumours has been the concept of 'malignant trophoblastic disease'. The term was introduced to overcome the fact that in certain patients treated by chemotherapy there was no histological diagnosis and, therefore, it was not known whether the patients had invasive moles or choriocarcinoma. Combined with evidence that 'early' treatment is better than 'late', and given the arbitrariness of the indications for chemotherapy after hydatidiform mole, the inexactitude of the concept precludes comparison of treatments, since it admits to the treated series patients with unproven mole and choriocarcinoma.

The treatment of $8 \%$ of patients with mole by chemotherapy is considered appropriate in the UK, and in N America about $20 \%$ are treated. Data from the prechemotherapy era indicated that about 2 to $3 \%$ of moles gave rise to choriocarcinoma and a further 2 to $3 \%$ to life-threatening invasive moles, so it has to be recognized that many patients are being treated either unnecessarily, or to preserve reproductive function rather than life.

Chemotherapy has come to be used for a highly heterogeneous group of patients. For many years our approach was to initiate it with a non-toxic methotrexate-folinic acid regimen and, then, if the response were inadequate, actinomycin $\mathrm{D}$ and, later, other drugs were used. This method has some advantages, but it now appears better to tailor treatment according to the risk both of disease and of treatment to the individual patient. At the same time another principle has emerged, though as yet it is less clearly defined: resistance to one drug, once it has become established, appears to reduce the response to other drugs. For this reason, sequential therapy starting with the less effective and less toxic forms of treatment and progressing towards multidrug therapy has given way to the policy of defining the likelihood of drug resistance at the start of treatment.

In our series to date we have used 30 different cytotoxic agents alone, 20 couplet combinations, eight triplet combinations, three quadruple combi- nations and eight multidrug combinations, radiotherapy, oestrogens, progestogens, androgens and various forms of adoptive, passive and active immunization. It will not be possible to review all these here but on the basis of the treatment used and its outcome it has been possible to define a series of prognostic factors by which the general pattern of treatment is determined. Some of these prognostic factors emerged partly in the early studies (Hertzet al, 1961).

In brief, it has been found (Bagshawe, 1976) that the prognosis deteriorates markedly the longer the interval between the end of the antecedent pregnancy and the start of chemotherapy, and it also deteriorates the higher the hCG values at the start of treatment. It might be thought that both factors relate to tumour volume but whilst this is probably true of hCG values it is clear that duration of disease is important and has an independent effect. Other important factors are whether the lesion is invasive mole or choriocarcinoma (the former having caused no deaths in our series) and the type of antecedent pregnancy (mole, non-mole abortion or full-term delivery) reflects this. The ABO groups of the patient and her husband also influence prognosis, the most unfavourable group being $\mathrm{AB}$ and $\mathrm{B}$ for the patient, followed by $\mathrm{A}_{q} \times \mathrm{O}^{x}$. Group $\mathrm{AB}$ and $\mathrm{B}$ husbands appear to confer a good prognosis. Metastases in the brain and to a lesser extent in the liver are unfavourable as are very large tumours masses at any site. A multiplicity of metastases is unfavourable unless these are small. Prognosis also deteriorates with increasing age and with increasing parity; the two effects may be independent and more than four antecedent pregnancies may be favourable, particularly in older women. Marked lymphocytic infiltration confers a better prognosis although there may be a small, biologically distinct type of tumour associated with gross lymphocytic infiltration and low hCG production which carries a bad prognosis. Patients who are immunosuppressed before chemotherapy have shown a poor therapeutic response.

A scoring system based on weighting these factors has been developed and applied retrospectively to our series. Patients in low score categories have had $100 \%$ survival and those in the highest have not survived. Summation of the various prognostic factors appears to operate strongly.

For practical purposes patients are allocated to three broad categories according to our scoring system. Patients in the low-risk category are mostly those with tumours treated within six months of evacuation of hydatidiform mole, and they are treated with four to seven courses of methotrexate $\left(30 \mathrm{mg} / \mathrm{m}^{2}\right.$ intravenously every 48 hours $\times 4$ with folinic acid $6 \mathrm{mg}$ intramuscularly 30 hours after each 
injection of methotrexate). Rest intervals between courses last seven days. This regimen does not cause alopecia; mucositis and vomiting are unusual and haemopoietic effects slight. It is a remarkable example of highly selective antitumour chemotherapy. The patient's treatment must, however, be carefully and continuously monitored by hCG measurements since it is disadvantageous to allow the tumours to continue to be exposed to an agent to which they are showing evidence of resistance. If resistance develops, of if the tumour recurs after remission has been obtained, then the patient is treated with 'medium-risk' type chemotherapy.

Patients with intermediate scores, and the $10 \%$ or so failing with 'low-risk' treatment, receive a regimen based on a series of alternating courses, with intervals of seven to 10 days between courses. Course $A$ is actinomycin $\mathrm{D}, 0.5 \mathrm{mg} / \mathrm{day}$, iv for five to seven days; course $B$ is vincristine $1.2 \mathrm{mg} / \mathrm{m}^{2}$ and cyclophosphamide $500 \mathrm{mg} / \mathrm{m}^{2}$ on days 1 and 3 ; course $C$ is methotrexate $30 \mathrm{mg} / \mathrm{m}^{2}$ im every 48 hours $\times 4$; folinic acid $6 \mathrm{mg}$ im 30 hours after each methotrexate injection; 6-mercaptopurine $75 \mathrm{mg} / \mathrm{m}^{2}$ by mouth daily on days 1 to 7 . If one of these regimens fails to produce a satisfactory response, course $\mathrm{D}$ which is adriamycin $30 \mathrm{mg} / \mathrm{m}^{2}$ and melphalan $6 \mathrm{mg} / \mathrm{m}^{2}$ on day 1 , is substituted for it.

Patients with the highest risk scores and those who fail to sustain remission after treatment with the intermediate score regimen are now treated by multidrug regimens which carry significant morbidity and some risk of fatality. Whenever possible the multidrug regimen is alternated with the less toxic intermediate score regimen. The current regimen for these patients includes hydroxyurea for 48 hours, followed by vincristine and moderately high-dose methotrexate for 24 hours followed by folinic acid for three days. Cyclophosphamide is given on the first day of folinic acid with actinomycin $D$ on the next three days. After a two-day drug-free interval adriamycin $30 \mathrm{mg} / \mathrm{m}^{2}$ and melphalan $6 \mathrm{mg} / \mathrm{m}^{2}$ are given provided generalized toxicity and haematological indices do not contraindicate. A prolonged high-dose multidrug regimen of this nature should not be attempted without full chemotherapy supportive services. The fact that such treatment is necessary from the outset for some patients to have a chance of achieving remission is one of the principal reasons for continuing to centralize the care of patients with trophoblastic tumours. Until 1973, no patient in our series in the highest risk categories had achieved sustained remission but since then, using multidrug regimen from the outset, some sustained remissions have been achieved.

Brain metastases present on admission have responded fully to treatment in $13 / 24$ patients. The development of brain metastases during treatment has been fatal in all but one case. Treatment has consisted of systemic chemotherapy with either intrathecal methotrexate or skull irradiation or both. Intrathecal methotrexate is given once or twice during each course of systemic chemotherapy. Although this appears advantageous it may introduce new problems. The danger of metastatic disease developing in the brain during chemotherapy has led us to a trial of prophylactic intrathecal methotrexate in patients with radiologically evident pulmonary metastases but this cannot yet be evaluated.

The changing treatment methods of the past 20 years have shown progressive improvement in the results achieved, particularly in patients in the intermediate risk category. It is interesting, however, that the use of screening procedures for patients with hydatidiform mole has not only limited unnecessary treatment but has resulted in earlier diagnosis. A significant part of the improvement in results can be attributed to the lower average 'scores' seen in recent years.

In the series to date, more than 100 women have had one or more normal pregnancies subsequently to treatment. Clinical and historical examination of 36 patients available for study has not revealed any residual abnormalities of hepatic or haemopoietic function. One patient has hypertension following methotrexate-associated renal failure. Their obstetric histories following chemotherapy were worse than those of a comparable control group but they were not worse than their own obstetric histories before chemotherapy (Walden and Bagshawe, 1976). The only second malignancy so far reported in the surviving patients, approximately 400 in all, is one cervical carcinoma in situ detected on routine cytology.

\section{References}

Bagshawe, K. D. (1969). Choriocarcinoma: The Clinical Biology of the Trophoblast and its Tumours. Arnold, London.

Bagshawe, (1976). Cancer (Philad.), in press.

Bagshawe, K. D., Golding, P. R., and Orr, A. H. (1969). Choriocarcinoma after hydatidiform mole: studies related to effectiveness of follow-up practice after hydatidiform mole. Brit. med. J., 3, 733-737.

Bagshawe, K. D., and Harland, S. (1976). Cancer (Philad.), in press.

Bagshawe, K. D., and McDonald, J. M. (1960). Treatment of choriocarcinoma with a combination of cytotoxic drugs. Brit. med.J., 2, 426-431.

Bagshawe, K. D., Orr, A. H., and Rushworth, A. G. J. (1968). Relationship between concentrations of human chorionic gonadotrophin in plasma and cerebrospinal fluid. Nature (Lond.), 217, 950-951.

Bagshawe, K. D., Wilson, H., Dublon, P., Smith, A., Baldwin, M., and Kardana, A. (1973). Follow-up after hydatidiform mole: studies using radioimmunoassay for 
urinary human chorionic gonadotrophin (HCG). $J$. Obstet. Gynaec. Brit. Cwlth., 80, 461-468.

Bohn, H. (1974). Studies on the pregnancy-specific- $\beta_{1}$ glycoprotein $\left(\mathrm{SP}_{1}\right)$. Arch. Gynäk., 216, 347-358.

Braunstein, G. D., Vaitukaitis, J. L., Carbone, P. P., and Ross, G. T. (1973). Ectopic production of human chorionic gonadotrophin by neoplasms. Ann. intern. Med., 78, 3945.

Brinck-Johnsen, T., Solc, J., and Galton, V. A. (1970). Urinary excretion of estrogens in women with hydatidiform mole and choriocarcinoma. Obstet. and Gynec., 36, 671-674.

Dawood, M. Y. (1975). Evaluation of serum progesterone during treatment of malignant trophoblastic disease. Amer. J. Obstet. Gynec., 123, 291-298.

Hershman, J. M., Higgins, H. P., and Starnes, W. T. (1970). Differences between thyroid stimulator in hydatidiform mole and human chorionic thyrotropin. Metabolism, 19, 735-744.

Hertz, R., Lewis, J., Jr., and Lipsett, M. B. (1961). Five years' experience with chemotheraphy of metastatic choriocarcinoma and related trophoblastic tumours in women. Amer. J. Obstet. Gynec., 82, 631-640.

Ishiguro, T. (1975). Serum a-fetoprotein in hydatidiform mole, choriocarcinoma, and twin pregnancy. Amer. J. Obstet. Gynec., 121, 539-541.

Jacoby, B. (1972). Studies on Placental Alkaline Phosphatases from Normal and Tumour Tissues. PhD Thesis, University of London.

Jacoby, B., and Bagshawe, K. D. (1972). A radioimmunoassay for placental-type alkaline phosphatase. Cancer Res., 32, 2413-2420.

Kardana, A., and Bagshawe, K. D. (1976). A rapid sensitive and specific radioimmunoassay for human chorionic gonadotrophin. J. Immunol. Method, 9, 297-305.

Kenimer, J. G., Hershman, J. M., and Higgins, H. P. (1975). The thyrotropin in hydatidiform moles is human chorionic gonadotropin. J. clin. Endocr., 40, 482-491.

Kock, H., Kessel, H. v., Stolte, L., and Leusden, H. v. (1966). Thyroid function in molar pregnancy. J. clin. Endocr., 26, 1128-1134.

Li, M. C., Hertz, R., and Spencer, D. B. (1956). Effect of methotrexate therapy upon choriocarcinoma and chorio- adenoma. Proc. Soc. exp. Biol. (N.Y.), 93, 361-366.

Lin, T. M., Halbert, S. P., Kiefer, D., Spellacy, W. N., and Gall, S. (1974). Characterization of four human pregnancyassociated plasma proteins. Amer. J. Obstet. Gynec., 118, 223-236.

Magrath, I. P., Golding, P. R., and Bagshawe, K. D. (1971). Medical presentations of choriocarcinoma. Brit. med.J., 2, 633-637.

Odell, W. D., Bates, R. W., Rivlin, R. S., Lipsett, M. B., and Hertz, R. (1963). Increased thyroid function without clinical hyperthyroidism in patients with choriocarcinoma. J. clin. Endocr., 23, 658-664.

Ross, G. T., Stolbach, L. L., and Hertz, R. (1962). Actinomycin $D$ in the treatment of methotrexate-resistant trophoblastic disease in women. Cancer Res., 22, 10151017.

Rushworth, A. G. J., Orr, A. H., and Bagshawe, K. D. (1968). The concentration of HCG in the plasma and spinal fluid of patients with trophoblastic tumours in the central nervous sytem. Brit. J. Cancer, 22, 253-257.

Samaan, N. A., Smith, J. P., and Rutledge, F. N. (1973). Plasma androstenedione (A) and dihydrotestosterone (DHT) in patients with trophoblastic disease and the effect of chemotherapy and oophorectomy. Gynec. Invest., 4, 237-242.

Samaan, N. A., Smith, J. P., Rutledge, F. N., and Barcellona, J. M. (1972). Plasma testosterone levels in trophoblastic disease and the effects of oophorectomy and chemotherapy. J. clin. Endocr., 34, 558-561.

Seppälä, M., Bagshawe, K. D., and Ruoslahti, E. (1972). Radioimmunoassay of alpha-fetoprotein: a contribution to the diagnosis of choriocarcinoma and hydatidiform mole. Int. J. Cancer, 10, 478-481.

Stone, M., Dent, J., and Bagshawe, K. D. (1976). In preparation.

Tatarinov, Y. S., Mesnyankina, N. V., Nikoulina, D. M., Novikova, L. A., Toloknov, B. O., and Falaleeva, D. M. (1974). Identification immunochimique de la bêta globuline de la 'zone de grossesse' dans le sèrum des malades atteintes des tumeurs trophoblastiques. Int. J. Cancer, 14, 548-554.

Walden, P. A. M., and Bagshawe, K. D. (1976). Amer. J. Obstet. Gynec., in press. 